Aleksandra Rajewska, Wioletta Mikołajek-Bedner, Joanna Lebdowicz-Knul, Małgorzata Sokołowska, Sebastian Kwiatkowski and Andrzej Torbé*

\title{
COVID-19 and pregnancy - where are we now? A review
}

https://doi.org/10.1515/jpm-2020-0132

Received March 26, 2020; accepted April 22, 2020; previously published online May 11, 2020

\begin{abstract}
The new acute respiratory disease severe acute respiratory syndrome coronavirus-2 (SARS-CoV-2) is highly contagious. It has caused many deaths, despite a relatively low general case fatality rate (CFR). The most common early manifestations of infection are fever, cough, fatigue and myalgia. The diagnosis is based on the exposure history, clinical manifestation, laboratory test results, chest computed tomography (CT) findings and a positive reverse transcription-polymerase chain reaction (RT-PCR) result for coronavirus disease 2019 (COVID-19). The effect of SARS-CoV-2 on pregnancy is not already clear. There is no evidence that pregnant women are more susceptible than the general population. In the third trimester, COVID19 can cause premature rupture of membranes, premature labour and fetal distress. There are no data on complications of SARS-CoV-2 infection before the third trimester. COVID-19 infection is an indication for delivery if necessary to improve maternal oxygenation. Decision on delivery mode should be individualised. Vertical transmission of coronavirus from the pregnant woman to the fetus has not been proven. As the virus is absent in breast milk, the experts encourage breastfeeding for neonatal acquisition of protective antibodies.
\end{abstract}

Keywords: coronavirus; COVID-19; delivery; pregnancy.

\footnotetext{
*Corresponding author: Prof. Andrzej Torbé, Department of Obstetrics and Gynecology, Pomeranian Medical University in Szczecin, Powstańców Wielkopolskich 72 Av, 70-111 Szczecin, Poland, Phone: +48914661466 , Fax: +48914661350 , E-mail: torbea@wp.pl Aleksandra Rajewska, Wioletta Mikołajek-Bedner, Joanna Lebdowicz-Knul, Matgorzata Sokotowska and Sebastian Kwiatkowski: Department of Obstetrics and Gynecology, Pomeranian Medical University in Szczecin, Powstańców Wielkopolskich 72 Av, 70-111 Szczecin, Poland
}

\section{History}

At the end of 2019, a new acute respiratory disease emerged, occurring in the very beginning in Wuhan city in Hubei province in China. Four untypical pneumonia cases were noticed on $26^{\text {th }}$ December 2019 in the Hubei Integrated Chinese and Western Medicine Hospital, and the day after were reported to the local Centre for Disease Control and Prevention (CDC). Due to its high contagiousness, the infection spread quickly reaching all continents and was declared by the World Health Organization (WHO) as a pandemic on $11^{\text {th }}$ March 2020. The aetiologic factor was identified as a coronavirus, belonging to RNA viruses, the genus of Betacoronaviruses, and named severe acute respiratory syndrome coronavirus-2 (SARS-CoV-2) [1]. With high probability the disease is a zoonosis, and its source was presumably Huanan Seafood Wholesale Market, where multiple species of animals, dead and alive, are being sold for catering purposes. Previously a coronaviral zoonosis, later known as severe acute respiratory syndrome (SARS), occurred in 2002, spilled out from Guangdong markets in China and was declared contained in July 2003. Also caused by Coronaviridae, Middle East respiratory syndrome (MERS) appeared in 2012 in Saudi Arabia and its outbreak continues to this day. The typical reservoir of coronaviruses are bat populations, from which the pathogen can be transmitted through the intermediate link like dromedary camels (MERS) or palm civets (SARS) to humans, achieving the ability of human-to-human transmission. SARS-CoV-2 seems to share the host receptor, which is angiotensin-converting enzyme-2 (ACE-2) with SARS-CoV, as it shares almost $82 \%$ of nucleotide sequences. Unlike SARS and MERS, despite a relatively low general case fatality rate (CFR), calculated at $2.3 \%$, SARS-CoV-2 is highly contagious, having caused much more deaths presented in absolute numbers. The main transmission route is via respiratory droplets, but a suggestion of alternative faecal-oral route also appears [2-4]. 


\section{Epidemiology}

As Zunyou et al. from the Chinese CDC report, until $11^{\text {th }}$ February 2020, 62\% of COVID-19 infections were confirmed by viral nucleic acid detection in pharyngeal swab samples. Twenty-two percent of the infections were defined as suspected cases, based on clinical symptoms and exposure history, due to insufficient availability of tests for rapidly increasing needs. Fifteen percent of diagnoses were made as "clinical" with the consideration of symptoms, exposure history and lung imaging results, without the use of nucleic acid tests. One percent of positive test result patients was asymptomatic. It is suspected that the real number of SARS-CoV-2 infections would remain unknown due to the inability to detect all asymptomatic and mild cases. Sixty-four percent of case clusters occurred in familial households, which shows the risk of viral transmission in close contacts.

Because there is no effective pharmacological treatment or vaccine, traditional methods must be implemented, including isolation, quarantine, social distancing and public transport limitations [5].

In the majority of patients (81\%), the course of the disease was mild, what is defined as non-pneumonic or mild pneumonia. Fourteen percent of severe cases were characterised by dyspnoea, tachypnoea, decreased blood oxygen saturation, drop of the ratio of partial pressure of arterial oxygen to fraction of inspired oxygen and/or massive lung infiltration. Only $5 \%$ of cases manifested critical severity like respiratory failure, septic shock and/ or multiple organ dysfunction. The general CFR was $2.3 \%$, with a clear distribution by age group, from no deaths among 9-year-old and younger children to $14.8 \%$ in patients over 80 years of age, who were considered as a high-risk group. Pre-existing comorbidities like cardiovascular diseases, diabetes, chronic respiratory disease, hypertension and cancer increased the CFR significantly. What is remarkable is that among $3.8 \%$ of cases in health workers as many as $14.8 \%$ were categorised as severe or critical [5].

\section{Diagnostics}

The diagnosis of SARS-CoV-2 infection is based on epidemiological exposure history, clinical manifestation, laboratory test results, chest computed tomography (CT) findings and a positive reverse transcription-polymerase chain reaction (RT-PCR) result for COVID-19. As Yang et al. accentuate, all these methods are simultaneously useful and limited, as even RT-PCR has a false-negative rate of at least $30 \%$, so the diagnostic process should include complex data. They recommend TOCC scheme: travel, occupation, contact and cluster history collection [1, 6-8].

The most common early clinical manifestations of infection are fever, cough, fatigue and myalgia, and sometimes headache and diarrhoea [7]. The International Society of Ultrasound in Obstetrics and Gynecology (ISUOG), basing on the WHO recommendations, addressed an interim guidance on coronavirus infection in pregnant women to healthcare professionals, containing, inter alia, clear and detailed classification criteria:

Suspected case:

- Acute respiratory illness and no other aetiology and travel or residence history in an area with local transmission of SARS-CoV-2 during 14 days before symptom onset

- Any acute respiratory illness and history of contact with a confirmed probable case in the 4 days prior to symptom onset

- Severe acute respiratory infection requiring admission to the hospital with no other aetiology

Probable case:

- Suspected case with an inconclusive laboratory test result

Confirmed case:

- Patient with a positive COVID-19 laboratory test result, at any clinical state, including those asymptomatic

The authors recommend to test every suspected case using molecular methods, especially viral RNA detection with quantitative RT-PCR (qRT-PCR) on lower respiratory tract specimens if available or on upper respiratory tract specimens in other circumstances. Lower respiratory tract specimens have a higher diagnostic value than upper respiratory tract specimens. The WHO recommends lower respiratory tract specimens, such as sputum, endotracheal aspirate or bronchoalveolar lavage, to be collected for testing. If it is not available, upper respiratory tract specimens of combined nasopharyngeal and oropharyngeal swabs should be taken.

Test should be repeated after at least 1 day in strongly suspected patients whose first test was negative [9]. Rassmussen et al. accentuate that not only upper and lower respiratory material and serum are relevant to test [7], other materials, including blood, urine and stool, can also be collected as enabling to follow the presence and the shedding of virus from different body compartments [7, 9]. Zhu et al., investigating the risk of maternal-fetal 
virus transmission, recommend taking samples of neonatal pharyngeal swabs, but also umbilical cord blood and amniotic fluid, and serum, gastric fluid, anal swabs or stool from the neonate [10].

In reports published so far, the criteria of recovery was not only subsiding clinical symptoms but also negative results of subsequent RT-PCR tests.

\section{Pregnancy}

The effect of SARS-CoV-2 infection on pregnancy is not already clear. Infection, especially viral pneumonia is a significant factor contributing to rising morbidity and mortality among pregnant women. The CFR for the pregnant women during the influenza pandemic in 19181919 was 27 to even 50\% if the exposure took place in the third trimester, while in the 1957-1958 Asian flu epidemic the CFR was twice as high for pregnant than for nonpregnant women [1].

Jenco and Yang et al. emphasise that pregnant women are generally in a high-risk group for infectious diseases due to gestational immunologic and physiologic changes in their system. According to former experiences with SARS, MERS and influenza, Schwartz states that pregnant women are more prone to develop severe pneumonia in case of respiratory pathogen infection than nonpregnant patients. Chronic or pregnancy-related comorbidities can increase the risk $[6,8,11]$.

As yet, there is no evidence that pregnant women would be more susceptible to COVID-19 infection or more prone to develop severe pneumonia than the general population [9]. Until December 2019 there were six coronaviruses prior known to infect people. Except Betacoronaviruses SARS and MERS, four of them, Alphacoronaviruses HCoV-229E and HCoV-NL63 and Betacoronaviruses HCoVOC43 and HCoV-HKU1, caused usually common cold symptoms and were able to cross the placental barrier [1]. Coronaviridae causing MERS and "classic" SARS affect pregnancy leading to adverse outcomes like miscarriages, fetal growth restriction, preterm labour and a significant rise in maternal mortality $[1,6,7]$. As Favre et al. suggest, as SARS-CoV-2 is in many aspects similar to those mentioned earlier, it is expected to have an analogous impact on pregnancy; however, in Yang et al.'s opinion, it seems to be less harmful for the pregnant women $[6,12]$.

Until now, the clinical manifestation of COVID-19 in the pregnant women seems to remain the same as in nonpregnant patients. It is a question of great importance whether the novel coronavirus can be transmitted vertically from the pregnant woman to the fetus. So far, the youngest infant tested positively for SARS-CoV-2 was a $36 \mathrm{~h}$ old [2]. There has not been proven maternal-fetal transplacental transmission of SARS or MERS coronaviruses so far $[6,11,12]$.

Liu et al. described 13 pregnant women, residing outside Wuhan city, with SARS-CoV-2 infection diagnosed with laboratory tests. Two of them were at less than 28 weeks of gestation, and 11 were in their third trimester. Seventy-seven percent presented fever, while only $23 \% \mathrm{had}$ dyspnoea and one was asymptomatic. Three patients were let home from hospital to continue pregnancy after having improved. All 10 women who stayed in the hospital had caesarean section, for emergency indications like fetal distress $(n=3)$, premature rupture of membranes $(n=1)$ and stillbirth $(n=1)$. The authors conclude that such perinatal complications could have been caused by coexistence of viral infection and pregnancy, beacause physiological changes in pregnancy reduce tolerance to hypoxia, especially in the third trimester. Six patients delivered prematurely between the $32^{\text {nd }}$ and $36^{\text {th }}$ week of gestation. One patient developed multiple organ dysfunction syndrome and septic shock, and required intensive care unit (ICU) admission, mechanical ventilation and eventually extracorporeal membrane oxygenation (ECMO) support [13].

Huang et al. ascribe particularly severe course of the disease to "cytokine storm", as they found a significantly higher cytokine concentration in patients who required ICU admission [14]. Chen et al. analysed nine pregnant women in the third trimester, admitted to the Zhongnan Hospital of Wuhan University presenting a positive result of RT-PCR test for SARS-CoV-2. They were aged from 26 to 40 years and were in 36 to $39+4$ weeks of gestation. One of them suffered from preeclampsia and one manifested gestational hypertension.

The clinical manifestation of SARS-CoV-2 varied among patients, but the most common symptom of all was fever developed before or even after delivery, then cough, myalgia, malaise, sore throat and shortness of breath, but also gastrointestinal symptoms occurred in one patient. There was no case of severe pneumonia with the need of mechanical ventilation. Laboratory tests found lymphopenia in five patients, elevated C-reactive protein level in six and increased concentration of aminotransferases in three of them. Lung CT scan showed in eight women multiple patchy ground-glass shadows, which were described as typical for the disease. In the group four preterm labours occurred. Two had premature rupture of membranes. Fetal distress occurred in another two. All nine women delivered by caesarean section, performed for different indications. What is important is that 
the researchers decided to notice COVID-19 pneumonia as a co-indication for surgical delivery [15].

The ISUOG interim guidance encourages to perform a chest CT scan in pregnant women with COVID-19 infection for diagnosis, especially in the situation of shortage of molecular tests, but also for the evaluation of patients' clinical condition. The authors accentuate that the radiation dose during single chest CT or even CT pulmonary angiogram is much lower than this causing fetal complications [9].

Weiyong et al. describe their experience with three pregnant women with SARS-CoV-2 infection acquired in the third trimester, confirmed with RT-PCR tests on throat swab samples. Two patients manifested fever and one complained of a cough. All of them had a typical chest CT scan picture, with changes characteristic of viral pneumonia. They report one case of meconium staining of membranes. The recovery process was followed with subsequent negative testing results for viral nucleic acid in samples [3]. Probably, like in the general population, it is impossible to identify asymptomatic patients among pregnant women. There are no sufficient data on pregnancy complications for COVID infection acquired before the third trimester. Zheng et al. found the expression of receptors of COVID-19 very low in maternal-fetal interface tissues, which can decrease the susceptibility for viral infection [16].

The ISUOG Guidelines emphasises that viral pneumonia of any aetiology in the pregnant women increases the risk of preterm birth, fetal growth restriction, perinatal mortality and low Apgar score in the neonate. As the consequences of COVID-19 infection in the pregnant women before the third trimester are not known, it is recommended to keep regular ultrasound fetal assessment every 2-4 weeks [9]. Zhu et al. presume that maternal hypoxia caused by SARS-CoV-2 can lead to fetal distress or premature labour, yet the authors themselves admit these are just speculations, due to poor evidence [10]. Wang et al. report a case of emergency caesarean section due to fetal demise in the $30^{\text {th }}$ week of pregnancy in $\mathrm{nCoV}$-19-positive woman, admitted to hospital and treated with Arbidol, lopinavir, ritonavir, cefoperazone and human serum albumin, with dexamethasone fetal maturation stimulation and magnesium sulphate prophylaxis, who gave birth to a healthy neonate, tested negatively for nCoV-19 [4].

Fetal maturity stimulation with steroid use should be avoided in critically ill patients for they can promote infection and worsen patient's condition. In case of spontaneous premature labour onset in the infected patient, tocolysis should not be administered to give steroids. Mehta et al. alarm that, besides acute respiratory distress syndrome (ARDS), the so-called cytokine storm can be another cause of mortality. The last is the colloquial name of secondary haemophagocytic lymphohistiocytosis, a hyperinflammatory condition characterised by fulminant hypercytokinaemia, often triggered by viral infections [17].

The ISUOG recommends pregnant patients to be treated in a tertiary hospital, with isolation of suspected or probable cases and negative-pressure isolation room stay for those confirmed. Medical staff should use personal protective equipment (PPE) including a respirator, goggle, face protective shield, surgical gown and gloves. Monitoring devices and ultrasound equipment must be disinfected carefully before another use. If such a care level is not available, in case of mild clinical manifestation, home confinement of patient should be considered [9].

\section{Delivery mode}

According to the ISUOG Guidance, iatrogenic preterm delivery should be considered by the multidisciplinary team individually in every case [9]. Weiyong et al. report two caesarean sections and one vaginal delivery in their patients [3]. The ISUOG Guidance recommends to allow to deliver vaginally if the onset of labour is spontaneous and its progress is satisfying, but the second stage should be shortened to avoid active pushing. Labour induced at favourable cervix should be intensified if the progress is poor, fetal distress develops or maternal condition worsens. Emergency caesarean delivery is necessary in septic shock, acute organ failure or fetal distress [9]. Yang et al. express their doubts whether women with COVID19 should be allowed to deliver vaginally, due to the risk of transmission of infection not only to the newborn, but also to medical professionals taking care of her during the labour. Vaginal labour, by its nature, can be a long-lasting process, with more time being spent by medical staff in close contact with the infected individual and increased exposure to respiratory droplets expired by the patient. Yet the authors declare that COVID-19 infection should not be the only indication for delivery and recommend individualised management, with patient's clinical state, gestational age and fetal condition taken into the account [6]. Wang et al. report a case of emergency caesarean section due to fetal demise in the $30^{\text {th }}$ week of pregnancy in $\mathrm{nCoV}$ 19-positive woman, who gave birth to a healthy neonate, tested negatively for nCoV-19 [4].

The COVID-19 infection itself should not be an indication for delivery, unless it is necessary to improve maternal 
poor oxygenation. Decisions should be individualised, considering maternal and fetal clinical conditions [9].

\section{Amniotic fluid}

Chen et al. did not find SARS-CoV-2 in the amniotic fluid collected from six infected women [15]. Weiyong et al. tested placenta and maternal vaginal mucus, finding them negative [3]. Wang et al. obtained virus-negative test results in the amniotic fluid, placenta and cord blood from an infected woman [4].

\section{Newborn}

Considering the vertical transmission of respiratory viruses from the mother to the child, there are three potential patterns: transplacental during pregnancy, via the birth canal during labour and by breastfeeding postpartum [10].

The evidence obtained until now suggests that vertical transmission of coronavirus from the pregnant woman to the fetus does not occur. Zhu et al. report nine newborns of mothers with COVID-19, manifesting a wide spectrum of symptoms like shortness of breath, fever, tachycardia or gastrointestinal dysfunctions, with chest radiography anomalies in seven of them. Two delivered prematurely developed severe complications, of which one of them died despite intensive treatment. All nine neonates were tested for viral nucleic acid presence in their throat swab specimens, and all of them were negative, which excluded the option of vertical SARS-CoV-2 transmission during pregnancy or delivery [10]. As Liu et al. report, among nine live born neonates of women infected with SARS-CoV-2, all got a 1-min Apgar score of 10 points. There was no clinical or serologic evidence for vertical virus transmission [13]. Chen et al. tested cord blood and throat swab from nine newborns of infected women, all with a negative result which seems to exclude SARSCoV-2 vertical transmission [15]. Weiyong et al. found cord blood and neonates' throat swab free from virus. They recommend to minimise neonate's exposure to maternal blood, using as obvious and simple procedure as washing neonate early after birth, and its isolation from the mother [3]. Wang et al. found negative results testing throat swab and gastric juice sampled from the neonate born to an infected mother [4].

A single neonate born on $6^{\text {th }}$ February 2020 was the first to be diagnosed to have SARS-CoV-2 as early as in
$36 \mathrm{~h}$ postpartum. Yet, the information is still insufficient as a potential evidence for intrauterine viral transmission due to the delay in throat swab collection made $30 \mathrm{~h}$ after birth.

\section{Breastfeeding}

Coronavirus has not been found in breast milk so far; so infected puerperal patients are recommended to maintain lactation. The tests performed by Chen et al. for coronavirus in the breast milk of nine women were all negative [15]. Based on the limited data collected so far, most researchers recommend breastfeeding or, at least bottle-feeding with expressed breast milk, as a potential source of maternal antibodies to be beneficial for the neonate; however, as Rassmussen et al. emphasise, protective antibodies to coronaviruses are, unfortunately, not long-lasting ones [6, $7,9,18]$. An infant should be bottle-fed by a healthy person, or, taking precautions, by its mother who must wear a face mask and wash her hands carefully to avoid transmission $[8,9]$. The ISOUG recommends that in asymptomatic or mildly affected cases breastfeeding and collocation are considerable, under the condition of maternal face mask use and careful handwashing [9].

\section{Treatment}

There is no specific treatment for SARS-CoV-2 infection. Typical proceedings include oxygen therapy, antibiotics, anti-inflammatory agents and antiviral therapy [3].

As COVID-19 can increase the risk of perinatal complications, Yang et al. recommend the management in a health care facility with close maternal and fetal monitoring, including early isolation, aggressive infection control procedures, oxygen therapy, avoidance of fluid overload, empiric antibiotics for possible bacterial superinfection, SARS-CoV-2 and co-infection testing, fetal and uterine activity control, early implementation of mechanical ventilation if necessary, individual delivery mode planning and multi-specialty consultations if needed [6]. As there is no specific treatment for COVID-19, the following are recommended:

- General management, including strict vital sign and oxygen saturation monitoring, repeated chest imaging, blood count, coagulation tests and renal and liver function assessment

- Symptomatic treatment: maintaining fluid and electrolyte balance, antipyretics 
- Fetal monitoring: cardiotocography in pregnancies over the $26^{\text {th }}-28^{\text {th }}$ week of gestation and ultrasound assessment of fetal growth and amniotic fluid volume with umbilical artery Doppler if needed

- Monitoring for bacterial secondary infection is recommended for confirmed cases, with the use of antibiotic treatment only if there is evidence of such complication

- In severe or critical disease, aggressive treatment is necessary, with, inter alia, hydration, oxygen therapy and chest physiotherapy; the management requires a negative-pressure ICU room for isolation and the support of interdisciplinary treatment

- In case of septic shock, fluid resuscitation and inotropic agents should be implemented, and the goal of oxygen therapy is to maintain saturation over $95 \%$ using an individualised method of ventilation $[3,6,9]$.

\section{Discussion and conclusions}

The most important limitation of this work is little data; however, its amount is rising day by day. Concluding from the former experiences, like SARS and MERS coronaviral infections, we should include pregnant women to a high-risk group for respiratory tract infections in general. Chen and Yang accentuate that immunologic suppression of pregnancy lead to increased susceptibility to infections, while physiologic changes like diaphragm elevation, respiratory tract mucosa oedema and increased oxygen consumption lead to poorer hypoxia tolerance $[6,15]$.

From another point of view, pregnancy seems not to increase the risk of severe course of nCoV-2019 infection, which differs from the "classic" SARS breakout, when even $50 \%$ of the pregnant women needed ICU admission, and as many as $33 \%$ required mechanical ventilation. So far, in available material, only one patient with nCoV-2019 infection developed severe pneumonia with the necessity of ECMO use. The infection seems to take the same pattern in pregnant and non-pregnant patients, but still we cannot exclude future trend changes [6].

The gold standard of diagnostics of SARS-CoV-2 infection is testing for viral nucleic acids. In reported patients, the most common symptoms were fever and cough, sometimes accompanied by myalgia, malaise, sore throat and shortness of breath. The most common laboratory test results included lymphopenia and elevated C-reactive protein concentration. Chen et al. emphasise that none of these findings are strictly characteristic of COVID-19 pneumonia, and recommend chest CT scanning as showing a typical picture of viral infection, with a high accuracy and a low false-negative rate [15].

Zhu et al. accentuate the importance of enhanced health education of society, effective prevention and appropriate patient triage as a collective effort to limit and then contain the outbreak [10].

Chen et al. performed caesarean sections in all nine patients, for different indications, including severe preeclampsia or previous C-section history, but they also admit to have taken into account the uncertainty of vertical virus transmission during uterine contractions and vaginal delivery [15].

As other SARS and MERS coronaviruses, the pathogen probably cannot cross the placental barrier, as no maternal-fetal transmissions were documented. Tests showed that cord blood, neonatal throat swabs and even breast milk from infected patients were free from the virus.

Yang et al. noticed that the interval from the clinical onset to delivery in reported patients did not exceed 7 days, so there is no evidence for vertical viral transmission risk in longer periods of pregnancy [6]. They recommend to allow for vaginal delivery only in case of spontaneous onset of labour, with a shortened second stage of labour. It seems necessary because, from one point of view, active pushing with the face mask on is difficult for the patient, while from another, forceful exhalation can reduce the effectiveness of mask and enhance exposure of medical professionals to viral transmission via the respiratory tract [6].

Till now there are no data on the influence of SARSCoV-2 infection on pregnancy in the first and second trimesters, especially on its potential to damage placental tissue and affect placental function. Careful observation, data collection and successive research are necessary.

Research funding: None declared.

Author contributions: All authors have accepted responsibility for the entire content of this manuscript and approved its submission.

Competing interests: Authors state no conflict of interest.

\section{References}

1. Schwartz DA, Graham AL. Potential maternal and infant outcomes from coronavirus 2019-nCoV (SARS-CoV-2) infecting pregnant women: lessons from SARS, MERS, and other human coronavirus infections. Viruses 2020;12:194. 
2. Wang L, Shi Y, Xiao T, Fu J, Feng X, Mu D, et al. Chinese expert consensus on the perinatal and neonatal management for the prevention and control of the 2019 novel coronavirus infection (first edition). Ann Transl Med 2010;8:47.

3. Liu W, Wang Q, Zhang Q, Chen L, Chen J, Zhang B, et al. Coronavirus disease 2019 (COVID-19)during pregnancy: a case series. Preprints 2020;2020020373.

4. Wang X, Zhou Z, Zhang J, Zhu F, Tang Y, Shen X. A case of 2019 novel coronavirus in a pregnant woman with preterm delivery. Clin Infect Dis 2020, doi: https://doi.org/10.1093/cid/ciaa200 [Epub ahead of print].

5. Wu Z, McGoogan JM. Characteristics of and important lessons from the coronavirus disease 2019 (COVID-19) outbreak in China. Summary of a report of 72314 cases from the Chinese Centre for Disease Control and Prevention. J Am Med Assoc 2020, doi: https://doi.org/10.1001/jama.2020.2648 [Epub ahead of print].

6. Yang H, Wang C, Poon LC. Opinion. Novel coronavirus infection and pregnancy. Ultrasound Obstet Gynecol 2020;55:435-7.

7. Rassmussen SA, Smulian JC, Lednicky JA, Wen TS, Jamieson DJ. Coronavirus disease (COVID-19) and pregnancy: what obstetricians need to know. Am J Obstet Gynecol 2020, doi: https://doi. org/10.1016/j.ajog.2020.02.017 [Epub ahead of print].

8. Jenco M. Experts discuss COVID-19 impact on children, pregnant women; AAP News. https://www.aappublications.org/ news/2020/03/12/coronavirus031220.

9. Poon LC, Yang H, Lee JC, Copel JA, Leung TY, Zhang Y, et al. ISUOG interim guidance on 2019 novel coronavirus infection during pregnancy and puerperium: information for healthcare professionals. Ultrasound Obstet Gynecol 2020;55:700-8.
10. Zhu H, Wang L, Fang C, Peng S, Zhang L, Chang G, et al. Clinical analysis of 10 neonates born to mothers with 2019-nCoV pneumonia. Transl Pediatr 2020;9:51-60.

11. Schwartz DA. COVID-19, SARS-CoV-2 and pregnancy: does the past predict the present?, https://www.contagionlive.com/.

12. Favre G, Pomar L, Musso D, Baud D. 2019-nCoV epidemic: what about pregnancies? Lancet 2020;395:e40.

13. Liu Y, Chen H, Tang K, Guo Y. Clinical manifestations and outcome of SARS-CoV-2 infection during pregnancy. J Infect 2020, https://doi.org/10.1016/j.jinf.2020.02.028 [Epub ahead of print].

14. Huang C, Wang Y, Li X, Ren L, Zhao J, Hu Y, et al. Clinical features of patients infected with 2019 novel coronavirus in Wuhan, China. Lancet 2020;395:497-506.

15. Chen H, Guo J, Wang C, Luo F, Yu X, Zhang W, et al. Clinical characteristics and interauterine vertical transmission potential of COVID-19 infection in nine pregnant women: a retrospective review of medical records. Lancet 2020;395: 809-15.

16. Zheng QL, Duan T, Jin LP. Single-cell RNA expression profiling of ACE2 and AXL in the human maternal-foetal interface. Reprod Dev Med 2020;4:7-10.

17. Mehta P, McAuley DF, Brown M, Sanchez E, Tattersall RS, Manson JJ, et al. COVID-19: consider cytokine storm syndromes and immunosuppression. Lancet 2020;395: 1033-4.

18. Dotters-Katz S, Hughes BL. Considerations for Obstetric Care during the COVID-19 Pandemic. Am J Perinatol 2020, doi: 10.1055/s-0040-1710051. [Epub ahead of print]. 